\title{
Efeitos de baixos números de Reynolds em um fluxo de canal turbulento totalmente desenvolvido
}

\author{
Andreão, W. L ${ }^{1 *} ;$ Feroni, R. C. ${ }^{2 \#}$ \\ ${ }^{1}$ Programa de Pós-Graduação em Saneamento, Meio Ambiente e Recursos Hídricos, Universidade Federal de Minas \\ Gerais, Belo Horizonte, MG, Brasil. \\ ${ }^{2}$ Departamento de Engenharias e Tecnologia, Universidade Federal do Espírito Santo, São Mateus, ES, Brasil. \\ e-mail: *w-andreao@ufmg.br ${ }^{*}$ rita.feroni@ufes.br
}

\begin{abstract}
Resumo
Uma simulação numérica utilizando o modelo de turbulência SST para um fluxo de canal turbulento foi realizada. As equações transientes de Navier-Stokes foram resolvidas no software Ansys CFX v16.0 para três números de Reynolds (70,110 e 180), baseados na velocidade de fricção e na metade da altura do canal. O perfil médio de velocidade e o coeficiente de atrito fluidodinâmico foram computados e comparados a estudos que utilizaram simulação numérica direta e ambos estão de acordo com a literatura citada. No perfil médio de velocidade foi observado regiões com comportamento linear e logarítmica. A influência do tamanho do domínio também foi avaliada para o número de Reynolds igual a 70 e sua influência nos resultados foi observada.
\end{abstract}

\begin{abstract}
A numerical simulation using the SST turbulence model for a turbulent channel flow was performed. The Navier-Stokes transient equations were solved in Ansys CFX v. 16.0 software for three Reynolds numbers (70, 110 and 180), based on the friction velocity and the channel half width. The mean velocity profile and the skin friction coefficient were computed and compared to studies using direct numerical simulation and both are in accordance with the cited literature. In the mean velocity profile, regions with linear and logarithmic behavior were observed. An influence of the domain geometry size on the results was also observed.
\end{abstract}

Keywords (Palavras chaves): SST, fluxo de canal turbulento, baixos números de Reynolds.

\section{Introdução}

A simulação de escoamento em canal turbulento totalmente desenvolvido tem sido realizada com ampla aplicação na área de mecânica dos fluidos. Quando o escoamento ocorre para baixos números de Reynolds, as estruturas coerentes tornam-se mais alongadas e estudos relacionados ao tamanho do domínio computacional adequado devem ser realizados. Estudos são vistos na literatura [1,2] onde atenção especial é dada em encontrar o menor domínio computacional possível que possa modelar corretamente a turbulência e também não aumente demasiadamente o custo computacional. Os autores utilizam em grande parte a correlação de dois pontos que deve tender a zero no centro do domínio para que seu tamanho seja satisfatório [1,2].

Uma das formas de se avaliar o escoamento turbulento é através do perfil de velocidade na direção principal do escoamento. O perfil turbulento bem desenvolvido próximo à parede é mostrado na Figura 1. A velocidade longitudinal adimensional, é dada por $U^{+}=u / u_{*}$ (onde $u_{*}$ é a velocidade de fricção, e $u$ é a componente de velocidade na direção longitudinal do escoamento), e a distância vertical adimensional a partir da interface é dada por $y^{+}=y u_{*} / v$ (onde, $y$ é a distância vertical a 
partir da interface e $v$ é a viscosidade cinemática do fluido).

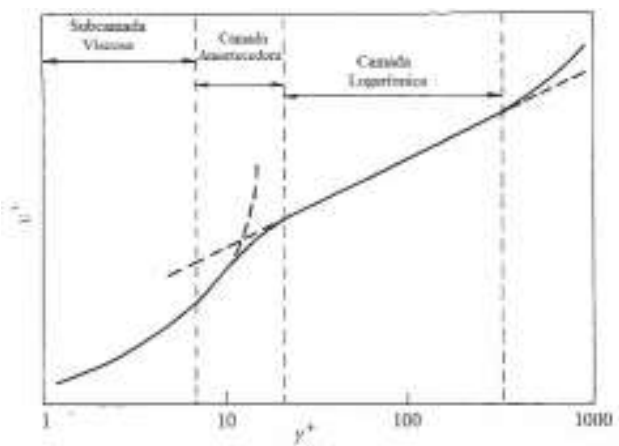

Figura 1: Configuração do perfil vertical de velocidade próximo à parede.

Fonte: Adaptado de [3].

De forma a tentar predizer os fenômenos e perfis turbulentos, são propostos modelos matemáticos com alto grau de sofisticação. Destacam-se os métodos: (i) baseados na decomposição de Reynolds, (ii) de simulação numérica direta (DNS) e (iii) de simulação das grandes escalas (LES).

Os modelos de turbulência baseados na decomposição de Reynolds são também conhecidos como modelos RANS (Reynolds Averaged Navier-Stokes), onde as variáveis das equações de Navier-Stokes são decompostas em uma parte média e outra flutuante.

Para modelos de uma e duas equações, são introduzidas equações de transporte adicionais para variáveis de turbulência, como a energia cinética de turbulência ( $k$ em $k-\varepsilon$ e $k-\omega$ ), a dissipação turbulenta $(\varepsilon)$ ou dissipação específica da energia cinética turbulenta $(\omega)$, por exemplo [4].

A maioria dos modelos baseados em $\omega$ são modelos de baixos números Reynolds por construção. Mas 0 modelo $k-\varepsilon$ padrão, e outros modelos $k-\varepsilon$ comumente encontrados, não os são. Os modelos de baixos números de Reynolds geralmente dão uma descrição muito precisa da camada limite. Os gradientes perto das paredes, no entanto, exigem resoluções de malha muito altas e que, por sua vez, significa um custo computacional maior. É por isso que métodos alternativos para modelar o fluxo próximo das paredes são frequentemente empregados para aplicações reais [4].

O modelo de turbulência Shear Stress Transport (SST) [5] é um modelo utilizado para baixos números de Reynolds e consiste numa combinação do modelo $k-\varepsilon$ no fluxo livre e do modelo $k-\omega$ perto das paredes. Para realizar isso, o modelo $k-\varepsilon$ é escrito em termos de $\omega$. Então o modelo $k-\omega$ e $\circ k-\varepsilon$ transformado são ambos multiplicados por uma função de mistura e somados. Sua formulação elimina alguns pontos fracos exibidos pelos modelos $k-\omega$ e $k-\varepsilon$ puros. Detalhes do equacionamento podem ser encontrados em [5]. Contudo, a malha computacional deve ser adequadamente refinada para capturar as características do escoamento próximo às paredes.

Nesse contexto, o objetivo do trabalho é aplicar o modelo de turbulência SST para caracterizar os fluxos de canais turbulentos totalmente desenvolvidos para três números de Reynolds (70, 110 e 180) e investigar se o tamanho do domínio computacional selecionado.

\section{Procedimentos numéricos}

Um esquema do domínio computacional é representado na Figura 2. Os experimentos numéricos foram realizados para os números de Reynolds ( $R e=u_{*} \delta / v$, sendo $\delta$ metade da altura do tanque) iguais a 70,110 e 180 . A Tabela 1 resume as dimensões do domínio para cada $R e$ a partir de Tsukahara et al. [1], que realizou o teste da correlação de dois pontos para garantir o tamanho mínimo da geometria simulada. $O$ tamanho da malha computacional, foi adotada a partir de um redimensionamento da malha utilizada por Tsukahara et al. [1]. Distância do primeiro volume de controle a partir das paredes $\left(\Delta x^{+}, \Delta y^{+}\right.$e $\left.\Delta z^{+}\right)$são idênticas a do estudo citado. As coordenadas e variáveis de fluxo são adimensionalizados por $\delta$, assumido igual a $0,018 \mathrm{~m}$, por $v$ e $u_{*}$. Condições periódicas foram assumidas nas direções longitudinal e transversal. Ainda na direção longitudinal, considerou-se um gradiente de pressão e um perfil de velocidade inicial para o desenvolvimento 


\section{Encontro Científico de Física Aplicada}

do escoamento. Nas paredes do fundo e interface foi considerado uma condição de não deslizamento.

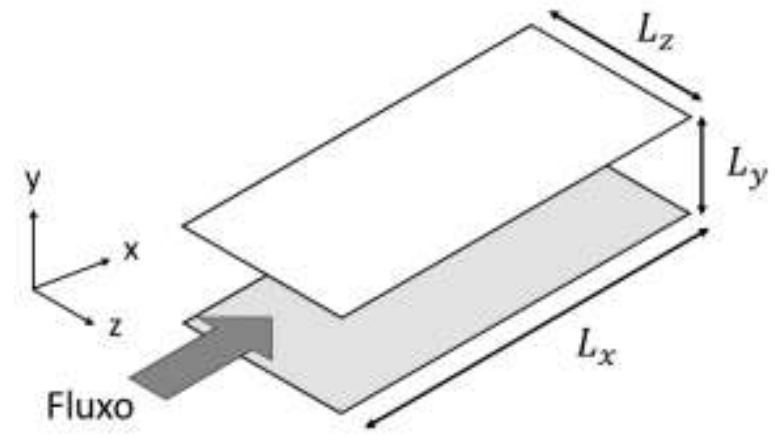

Figura 2: Representação do domínio computacional.

\begin{tabular}{cccc}
\multicolumn{4}{c}{ Tabela 1: Parâmetros da modelagem numérica. } \\
\hline Parâmetros & $\operatorname{Re~70}$ & $\operatorname{Re~110}$ & $\operatorname{Re~180}$ \\
\hline$L_{x} \times L_{y} \times L_{z}$ & $0,46 \times 0,036 \times$ & $0,23 \times 0,036 \times$ & $0,23 \times 0,036 \times$ \\
& $0,23(\mathrm{~m})$ & $0,115(\mathrm{~m})$ & $0,115(\mathrm{~m})$ \\
\hline$N_{x} \times N_{y} \times N_{z}$ & $128 \times 44 \times 64$ & $128 \times 44 \times 64$ & $128 \times 44 \times 64$ \\
\hline$\Delta x^{+}$ & $0,0036(\mathrm{~m})$ & $0,0025(\mathrm{~m})$ & $0,0018(\mathrm{~m})$ \\
\hline$\Delta y^{+}$ & $0,00004(\mathrm{~m})$ & $0,00005(\mathrm{~m})$ & $0,00004(\mathrm{~m})$ \\
\hline$\Delta z^{+}$ & $0,0018(\mathrm{~m})$ & $0,0012(\mathrm{~m})$ & $0,0009(\mathrm{~m})$
\end{tabular}

O software Ansys CFX v.16.0 [8] foi utilizado para resolver as equações de conservação da massa e quantidade de movimento. O escoamento foi assumido transiente, isotérmico e o modelo de turbulência SST foi utilizado. O critério de convergência utilizado foi um resíduo médio normalizado igual a 5e-6. Os resultados foram validados com a simulação numérica direta (DNS) realizada por Tsukahara et al. [1] para o $\mathrm{Re} 70$.

\section{Resultados}

O coeficiente de atrito fluidodinâmico $\left(C_{f}\right)$, definido pela Equação 1, é apresentado na Figura 3 para os diferentes números de Re.

$$
C_{f}=\tau_{w} /\left(\frac{1}{2} \rho u_{m}^{2}\right)
$$

onde $\tau_{w}$ é a tensão de cisalhamento na parede e $u_{m}$ é a velocidade média do seio da massa líquida, que foi calculada com base na metodologia proposta por Calmet e Magnaudet [6].

Os valores de $C_{f}$ foram comparados aos obtidos por Tsukahara et al. [1], Kim et al. [2] e Abe et al. [7], sendo estes dois últimos apenas para o $\operatorname{Re} 180$. Os coeficientes encontrados estão de acordo com aqueles mostrados pelos autores anteriores, apresentando decréscimo com o aumento do Re.

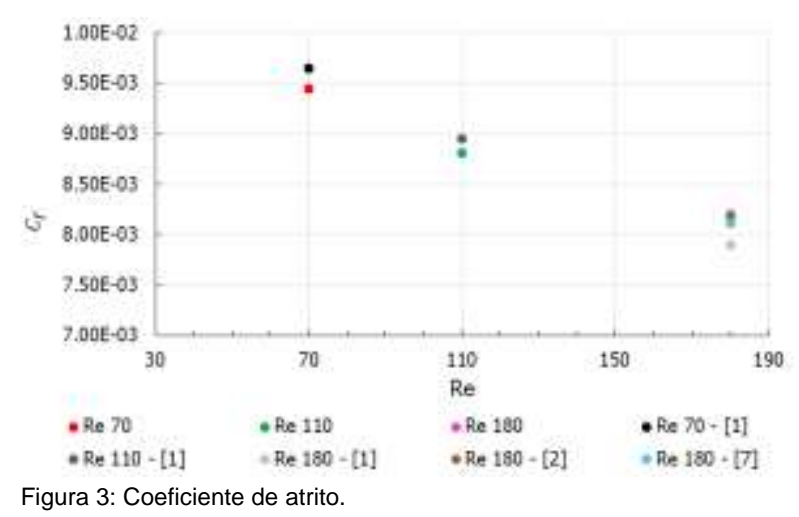

O perfil médio de velocidade na direção longitudinal é mostrado na Figura 4 para os três Re testados, e comparado com o resultado obtido por Tsukahara et al. [1]. O perfil de velocidade para $\mathrm{Re}$ igual a 70 do presente estudo obteve uma boa concordância com o perfil de Tsukahara et al. [1], com maior erro pontual sendo de $8 \%$.

Os perfis para todos os $\mathrm{Re}$, possuem uma região em que o comportamento é linear (até $y^{+} \approx 11$ ). A partir de $y^{+}>30$, tem-se a camada logarítmica, na qual os efeitos turbulentos são mais significativos. Percebe-se que o perfil para Re igual a 70 não evidencia muito a região logarítmica como os demais casos, sugerindo um regime de escoamento próximo ao laminar. Nota-se também que, para Re 70 , o perfil de velocidade está deslocado para cima, sugerindo que a espessura da subcamada viscosa se torna maior que para os outros casos.

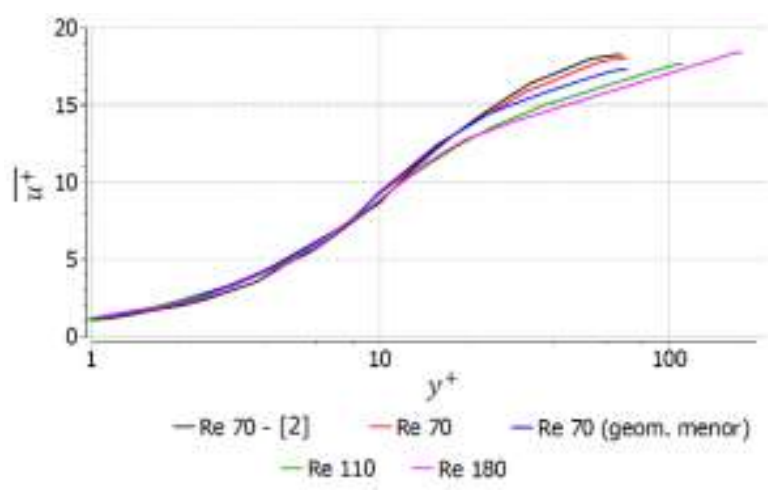

Figura 4: Distribuição média de velocidade. 


\section{Blucher Proceedings \\ VIII Encontro Científico de Física Aplicada}

\section{Blucher}

A fim de avaliar a influência do tamanho da geometria, para o Re igual a 70 também foi considerada uma simulação em que a geometria possui tamanho igual ao dos demais Re (110 e 180), ou seja, menor do que a proposta por Tsukahara et al. [1]. Esta modelagem apresentou valores de velocidades médias menores para maiores valores de $y^{+}$. Provavelmente, o perfil não se desenvolve corretamente, uma vez que as estruturas turbulentas mais alongadas estão confinadas em uma geometria menor do que a ideal.

\section{Referências}

[1] TSUKAHARA, T. et al. In Proceedings of the 4th International Symposium on Turbulence and Shear Flow Phenomena, Williamsburg, VA, p. 935-940, 2005.

[2] KIM, J. et al. J. Fluid Mech., v. 177, p. 133-166, 1987.
[3] DAVIDSON, P. A. Turbulence: an introduction for scientists and engineers. Oxford: Oxford University Press: 2009.

[4] Versteeg, H. K.; MALALASEKERA, W. An Introduction to Computational Fluid Dynamics: the finite volume method. Essex: Longman Scientific \& Technical: 1995.

[5] MENTER, F. R. AIAA Journal, v. 32, p. 1598-1605, 1994.

[6] CALMET, I.; MAGNAUDET, J. J. Fluid Mech., v. 474, p. 355-378, 2003.

[7] ABE, H. et al. J. Fluids Eng., v. 123, p. 382-393, 2001.

[8] CFX Ansys - Ansys Inc, 2014. 